\title{
Responsibly Managing Risks To The Environment: Stakeholders And Their Communication Contract
}

\author{
Birgitta Dresp-Langley*
}

\author{
Centre National de la Recherche Scientifique CNRS UMR 5508 University of Montpellier France
}

\begin{abstract}
The design of tools and procedures for the responsible and effective management of risks to humans and their environment is an important topic in modern environmental engineering. This article places the ethical ground clauses of a communication contract in the particular context of early hazard warnings. How respecting ethical ground clauses of communication may help avoid that the short-term economic interests of a few are placed before the long-term interests of society as a whole is explained on the basis of examples from disaster case studies. The need for rules which ensure that relevant information is effectively transmitted, received, and taken into account promptly is highlighted. Why successfully implementing such rules involves the individual responsibility of all stakeholders, from witnesses or victims to scientific experts and policy makers, is made clear. The ethical ground clauses of the communication contract introduced here provide universal rules for responsible communication, defined in terms of general guidelines for sincere, transparent, prompt, and cooperative information sharing, in particular in risk management. Earlier work has shown that implementing such a communication contract in corporate decision making helps promote stakeholder responsibility awareness, and triggers a learning process for initiating and fostering individual and collective behavior that will ultimately lead to responsible decisions and actions. These latter are the prerequisite for mitigating the potentially disastrous consequences of non-action in response to early warnings, when relevant scientific data and/or expert knowledge are not adequately taken into account because of faulty communication, identified as the major cause of delayed action in numerous case studies. Limitations of the communication contract theory are pointed out.
\end{abstract}

Keywords: Environment - Risk Management - Early Warnings - Policy making - Social factors - Communication Contract.

\section{INTRODUCTION}

Modern environmental engineering is to deliver technological solutions for the most pressing problems of society, and the research, theory, and practice in this still new and expanding field are evolving fast. Beyond scientific knowledge that helps developing strategies for a more efficient use of resources, the field needs theoretical frameworks from the human and economic sciences to generate global understanding of problems of uncertainty and instability in the evaluation and management of hazards such as natural disasters. How large-scale risks, ahead of and beyond early stages of warning, can be managed effectively and responsibly to protect the well-being of humans and their environment, and how to deal with the exposure and vulnerability of individuals or communities to multiple and often interrelated hazards represents a major challenge.

Stakeholder theory, which relates to social contract theory $[1,2]$, proves a powerful theoretical tool in hazard management from the earliest stages of alert to the final instances of decision and policy making. It can be used to address

*Address correspondence to this author at the Centre National de la Recherche Scientifique CNRS UMR 5508 University of Montpellier France; Tel: 00334671445 33; Fax: 00334671445 55;

E-mail: bdrespla@univ-montp2.fr some of the social and managerial core issues relative to problems of vulnerability and responsibility. Any individual, group, or community directly or indirectly, passively or actively, involved in or affected by an activity or project incurring risks becomes a legitimate stakeholder. Yet, identifying and characterizing stakeholder identity is not self-evident. There are the potentially exposed, and therefore vulnerable stakeholders on the one hand, and the stakeholders who are to be responsible for taking care of this vulnerability on the other. Fully respecting all the potential stakeholders is particularly important at the earliest stages of risk management, especially when the health and safety of human individuals and communities is at stake. Priority is to be given to making sure that who and what matters most is accurately identified as soon as possible. The present article introduces the concept of a communication contract [4] between stakeholders and/or their proxies, based on core concepts from social contract theory and communication theories and placed in the context of early multi-hazard risk management. The communication contract [4] permits to clarify stakeholder rights and responsibilities at several major levels of risk analysis.

Social and psychological factors act to either dampen or amplify both the collective and individual perception of risk in a given context [5]. Cross-cultural differences need to be considered for assessing such perception and its potential, negative or positive, influence on decision making and regu- 
latory action. The communication contract provides a learning tool for increasing awareness of both individual and collective responsibilities in crisis management, as testified by the results from the initial case study [4], where the conceptual framework of this theory was implemented in the decision making process of a small organization. The ground conditions (clauses) of the communication contract are universal and based solely on principles of common sense, independent of hierarchical status, culture or education level. The respect or non-respect of the clauses by members of a group or organization, however small or large it may be, can be straightforwardly monitored by any individual with a minimum of training to ensure responsible information sharing, decision making, and action from the earliest instances of risk scenarios that are likely to affect man, the environment, or any critical cycle upon which life depends. At such early instances, data and/or expert/witness knowledge relative to exposure and vulnerability are generally available, but too often not adequately taken into account.

\section{ASSESSING EXPOSURE AND VULNERABILITY TO HAZARDS}

Apart from providing descriptive statistical data, in terms of hazard indexes or severity metrics such as number of deaths or loss in income, risk analysis and management is to optimize hazard preparedness and to help design hazard mitigation strategies [3]. This requires a global systems perspective that recognizes the complex interactions between environment and social systems, at local and global levels of human aggregation. For a definition of the notion of "risk" consistent with such a perspective, we may propose that a group, community, area, region, or environment is to be considered "at risk" whenever there is a high probability of adverse impact from one or more hazard events. The inherent heterogeneity across multiple hazard scenarios often does not permit suggesting a single measure or even globally consistent multiple measures of hazard severity in complex situations. Meaningful quantitative estimates of random or systemic uncertainties and other quantitative analyses are often compromised by a substantial lack of knowledge about hazard occurrence and loss in specific time frames. Since such knowledge is, however, critical for putting into place mitigation policies, quantitative risk analysis for timely decision making has become a hot topic in current research programs worldwide.

Artificial intelligence, machine learning approaches and computational modeling using fuzzy logic and genetic algorithms have made it possible to simulate and predict critical changes in the environment with great precision in space and time [6-11], yet, whether the scientific data are effectively taken into account for adequate decision making and action depends on other, social, economical, and psychological, factors. Also, some hazards may have adverse local impacts on regions and communities and other, essentially positive, effects at a larger scale. This would be the case of thunderstorms, devastating coastal regions and affecting populations locally, but engendering globally beneficial effects on agriculture and water resources, for example. Apart from such particular and exceptional scenarios, the consequences of hazardous events, such as death or injury of people or loss of valuable structures and assets, are generally undesirable, and very often detrimental to many more than those directly affected. This is one of the reasons why the concepts of 'exposure' and 'vulnerability' are core issues in early warning systems for understanding the multiple implications of the notion of "risk", which is associated with a wide range of natural and man-induced hazards.

Assessing the degree of exposure of humans and their environments to different potential hazards is anything but straightforward, and decision makers are generally afraid of overreaction to what could be a "false positive". Moreover, accurate and reliable probability estimates for population exposure to specific types of hazard and for a whole range of event magnitudes and characteristics are difficult or impossible to achieve. Population distributions and activities vary across time and are governed by a multitude of socioeconomic factors and variables. The differential rates of such change cannot straightforwardly be projected into the future. Also, the stresses to which any given element at risk is subjected to will depend on hazard magnitude and other characteristics. Such stresses include mechanic solicitation in the case of earthquakes, inundation in the case of floods, and so forth. Any given element at risk may be extremely vulnerable to one hazard and unaffected by another, as some buildings may, for example, collapse under seismic stress and incur damage through thunderstorms and floods, but suffer very little or no stress during a drought. For any given hazard, vulnerability will vary from one element to another, as houses erected on platforms, for example, may be less vulnerable to flooding than other houses within the same area. Individuals and communities with larger resources and economic alternatives tend to be less vulnerable to, and able to recover more quickly from, the stresses and damages incurred by a hazard than populations with fewer resources.

\section{THE VULNERABILITY VERSUS RESPONSIBIL- ITY DILEMMA}

Regulations for adequate action are needed just as much as quantitative predictions and statistical data characterizing hazards in terms of consequences on individuals and communities and their social and economic activities. However, making information about risk available to as many individuals as possible often happens too late. International regulations and recommendations for the handling of chemicals, for example, now worldwide recognized as dangerous to human health and the environment, only exist since 2002, such as the GHS recommendations of the United Nations for safe production, transport, and use, or the REACH regulations of the European Commission, with national helpdesks for the different countries, which exist only since 2007. Individual and collective differences in risk perception have been identified as important social and psychological variables, which ultimately affect regulative decision making and action in any society [5]. Dealing with risks effectively and responsibly requires overcoming this problem. Vulnerable stakeholders and those who are responsible to protect them from exposure need to be clearly identified, and all stakeholders are to be made aware of their individual responsibilities in the underlying communication process, regardless of their social status, education, or culture. This requires an effective system of information exchange between analysts, policy makers, experts, witnesses and other members 
of the public. What the policy makers need most, beyond scientific evidence or expert data, are sound guidelines which enable them to make the right decision at the right time.

Social contract theory [1] stipulates that we agree as individuals within society to adhere to an implicit contract, a so-called social contract. Through this social contract, we gain rights by giving up unlimited freedom and by accepting to respect and defend the rights of others. The idea that all rational beings would inevitably consent to such a social contract because it is in their own best interest was first introduced in theoretical essays by philosophers in the seventeenth and eighteenth century. Contemporary stakeholder theory originates from these writings and Donaldson and Dunfee's [2] integrative social contract theory has substantially contributed to the success of this theoretical stream by providing a fresh conceptual framework for ethical management in modern society. The term "integrative", places emphasis on the general, all-encompassing nature of the social contract as a basic commitment with binding obligations, which imply adhering to certain ethical core values and respecting certain rules of due process. Ethical core values are, in principle, collectively acknowledged though not always explicitly formulated, and can be considered universal in the sense that they are detached from specific cults, religions or beliefs. Beneficial to society in general, and to any individual who is part of it in particular, they are non-negotiable. They are the foundations of ethical standards in modern society, and almost invariably include: responsibility, integrity, honesty, respect, trust, openness, fairness and transparency. Translating ethical core values into action requires an explicit system of ground rules and principles. Such principles are particularly important when it comes to dealing with individuals, communities, or societies exposed to, and therefore vulnerable to, risks. The vulnerability-versusresponsibility dilemma describes the fact that vulnerable stakeholders and decision making instances often have conflicting interests. It can only be resolved by putting an emphasis on individual stakeholder responsibility at all levels of decision making and by placing the protection of the potentially exposed before other goals, such as securing the shortterm financial benefits of avoiding early actions to early warnings, for example. Involving individual stakeholder responsibility at all levels, from early warning analysis to the decision and policy making process, clarifies the ethical grounds of risk engineering and management prior to legal or economic implications.

Modern risk analysis aims at global systems approaches and integrative knowledge generation for a deeper understanding of the risks posed by multiple and often interdependent hazards [3]. However, from a purely technical viewpoint such global system approaches have thus far had very little impact on management and policy making. Exposure and vulnerability estimates are almost always based on relatively limited amounts of data, and the social and economic consequences of hazards, such as mortality, disease, or impoverishment due to resource losses, are evaluated at best in form of national statistics.

Dilley's [3] model, published by the World Bank Press a few years ago, defines a minimum of six universal steps

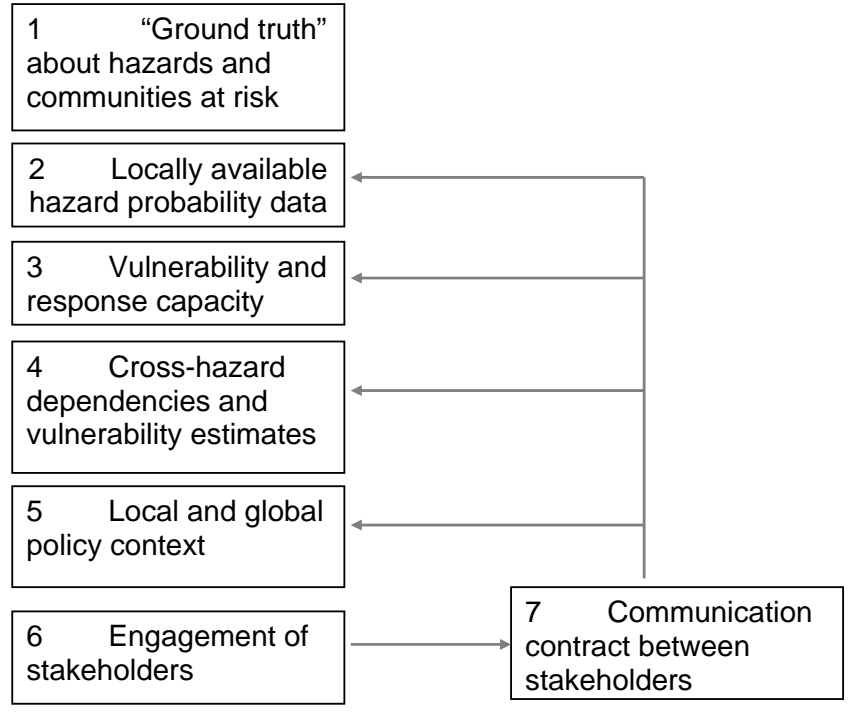

Fig. (1). A multi-hazard risk management model inspired from Dilley, 2005 [3]. The outcome of the communication process between stakeholders at levels 5 and 6 has impacts on all levels of data processing and information sharing.

(Fig. 1) in multi hazard risk management. The first model stage describes what may be called the "ground truth" (level $1)$. This refers to the true state of matters regarding exposures to multiple-hazard risks of regions worldwide. This true state will never fully be known because it cannot be reliably assessed because of technical and practical limitations to generating the necessary data, as explained above. Early warning systems rely on hazard probabilities and estimates of vulnerability and response capacity sampled by experts independently at levels 2 and 3 . Individual stakeholder responsibility comes into the game at these and all further levels, although the earliest stages do not involve all of the stakeholders. Simulations of cross-hazard dependencies and their interaction with other vulnerability estimates are provided at level 4 . The data patterns are then to be examined in the light of currently existing policies and measures to enable decisions about whether they are adequate or not, and which other measures are likely to be necessary (level 5). Whenever such a critical decision making process is triggered, all stakeholders need to be fully informed, and given specific tasks and responsibilities (level 5 and 6).

The quality and reliability of information exchanges between all levels of the model directly determines "who knows what and when" in risk management. This clarifies why level 7 of the risk analysis model considered here ultimately controls all prior levels of analysis. Early warning systems are useless, if they do not lead to proper action. However reliable early signs of alert may be, they are consistently more likely to trigger action in terms of appropriate mitigation strategies if they are known by as many stakeholders as possible, whether these are experts or not, and whatever the nature of the risk (floods, storms, forest fires or other). Whether relevant data regarding risks are communicated in due course to as many stakeholders as possible entirely depends on individual risk awareness and responsibility. 


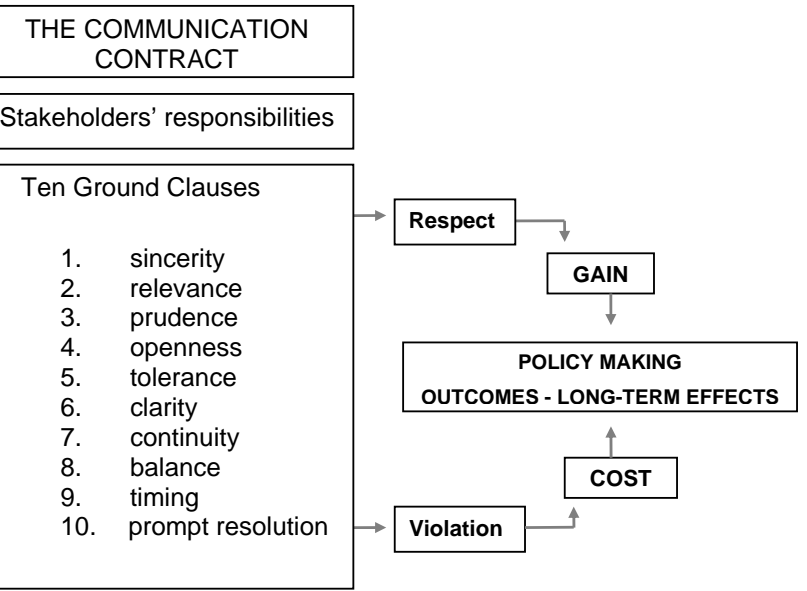

Fig. (2). Responsible communication for the management of hazard warnings at the earliest possible level of alert is placed under the premises of a communication contract.

\section{THE COMMUNICATION CONTRACT}

Transparent and responsible communication for the most effective information selection and knowledge sharing possible under conditions given, can be ethically and pragmatically placed under the premises of a communication contract, with explicit ground rules and clauses for due process, bearing in mind that communication is the most essential medium for promoting ethical core values in modern management. As will be made clear later herein, top heavy decision making hierarchies in policy making are generally a hindrance to putting ethical core values into action, mainly because the communication process is either too slow or fails completely.

The recognition of ethical aspects of communication stems from speech act theory $[12,13]$, which considers that the spoken or written word is equivalent to action, with similar implications and consequences. The Scottish philosopher Thomas Reid, one of the founders of the School of Common Sense Philosophy, was the first to explicitly state the nature of communication acts which involve individual moral responsibility (moral agency). In his essays on the active powers of the human mind [14], Reid points out that we enter a social contract, which we are expected to respect, whenever we ask, testify, command, or make promises. Reid's philosophy clarifies why the notion of a communication contract, follows directly from that of a social contract. Society and any group or organization that is part of it is defined as a community of communicating individuals who agree to adhere to an implicit communication contract. Through such a communication contract, individuals gain rights by giving up unlimited freedom, and by accepting to respect the needs, freedom, and rights of others. In the global corporate world, the growing trans-national embedding and interdependence of complex issues such as life quality, environmental challenges, economic development and sustainability have increased the need for individual social responsibility. To address these issues, contemporary sociological theories [15] inspired by Reid's philosophy place human agency at the centre of any future capacity of control over the nature and quality of all forms of human existence, from families to entire nations. Responsible communication between indi- viduals has undeniably become one of the most urgent of all current social needs, worldwide.

The clauses of the communication contract (Fig. 2) encompass and extend Austin's felicity conditions [12] by adapting them to the communication needs of modern society and management in particular. As ground clauses for ethical communication, they aim at exercising control over any severe form of non-execution or abuse of Austin's felicity conditions. On the basis of no more than ten ground clauses, the communication contract ensures successful communication at all levels of information exchange.

The sincerity clause is the conditio sine qua non of all ethical and responsible communication. The quality and reliability of policy management stands and falls with this clause, especially when the probability of risk is high and impacts beyond tangible material limitations are to be expected. The communication contract is based on the assumption that the sincerity clause is, for the most essential, respected by all partners involved because it is in their own best interest to do so. Starting from there, nine additional clauses regulate information exchanges for optimal decision making by ensuring the following conditions:

$\checkmark$ the most relevant information is given priorityrelevance clause

$\checkmark$ uncertain information is handled prudently-prudence clause

$\checkmark$ information is communicated openly and transparently - openness clause

$\checkmark$ differences in viewpoint are respected and discussed - tolerance clause

$\checkmark$ information is communicated transparently avoiding jargon - clarity clause

$\checkmark$ information sharing is ensured on a regular basiscontinuity clause

$\checkmark$ information sharing is balanced-balance clause

$\checkmark \quad$ information is shared as it becomes available-optimal timing clause

$\checkmark$ necessary decisions are not to be delayed-prompt resolution clause

In the context of decision and policy making for the implementation of early warning systems, the clauses of the communication contract provide a checklist for responsible management. It helps avoiding that what is known by a few is not shared by others, that potentially important information, data, or conclusions are dismissed on the basis of hierarchical considerations, or that the jargon employed by some experts hinders effective decision making by stakeholders in charge who themselves are not experts, for example.

\section{LATE ACTION TO EARLY WARNINGS: BETTER SORRY THAN SAFE?}

The consequences of non-communication and late action in cases where early hazard warnings were ignored or dismissed reveal the full extent of the vulnerability-versusresponsibility dilemma and highlight the need for a clear and 


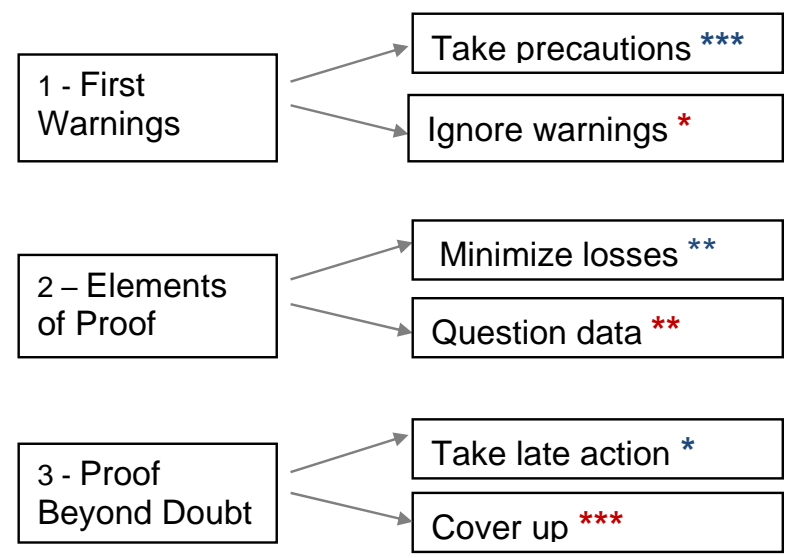

Fig. (3). Three levels of hazard proof need to be considered. Ignoring early warnings at the first level of early alert is likely to induce latent periods of non-action between first exposures (level 2) and late damages (level 3). This may produce cascades of irreversible consequences before any action can be expected to get things under control. Disaster levels (red asterisks) induced by communication failures leading to non-action increase with the level of proof, while the level of control through action diminishes with increasing level of proof (blue asterisks).

firm communication contract between stakeholders. Failure to communicate, decide, and act whenever early warnings are issued can have disastrous consequences, as illustrated by international case studies published by the European Environment Agency (EEA) [16]. To give just two examples here: the damages caused by long-term exposures to antimicrobials in food animals [17], or to blue asbestos dust in factories [18] extend over scales which nobody seemed to have anticipated when the first hazard warnings were issued. A closer look at the other thirteen case studies reveals that in all cases

- Early warnings were generally dismissed or ignored

- Action was triggered only when proof beyond reasonable doubt was available and most often far too late

At this late stage, the lack of communication and adequate decision making at earlier stages had already taken its toll and incurred damages and economic costs producing snowball effects across communities and nations. The statistics [16] reveal truly disastrous scenarios and incommensurable losses. While family doctors give the benefit of doubt to their patient by deeming that it is better to be safe than to be sorry, policy making for risk prevention seems to obey the opposite rule.

What needs to be communicated to whom and when and how early action should be taken needs to be clarified and implemented in a communication contract for hazard management. Three different levels of warning and certainty need to be considered (Fig. 3).

The first level describes a situation where early warnings are formulated and where the first preventive actions should be taken. Case histories have revealed that such early warnings are generally ignored or dismissed by policy makers because the short-term financial cost of reacting to what is deemed a potentially false positive is to be avoided. For ex- ample, the very first early warnings of asbestos induced health hazards were formulated in 1898 by a Woman Medical Inspector of the Crown, who instigated microscopic analyses of the sharp, jagged glass-like asbestos dust particles and concluded on their damaging effect on bronchial tubes and lungs. Her conclusions were confirmed a few years later by similar reports of two other Women Inspectors, published in the annual reports of Her Majesty's Chief Inspector of Factories in the UK, yet, the available evidence was not communicated to all the stakeholders. The politicians who were aware of the reports at the time disregarded them completely and by doing so, they were giving priority to shortterm economic gains to the detriment of long-term sustainability and human health. This case scenario is an example of a violation of ground clauses 2 (relevance), 4 (openness), 8 (balance), 9 (optimal timing) and 10 (prompt resolution) of the communication contract. Highly relevant data indicating potential risk to public health from several competent reports were ignored or dismissed by a few irresponsible stakeholders when these data should have been given priority (clause 2), communicated as soon as possible (clause 9) to as many stakeholders as possible (clause 8 ) and discussed by a balanced panel of several independent experts (clause 8) to ensure that, should action be deemed necessary, it can and will be taken promptly (clause 10). Which rules and procedures for proper communication, decision making and action could have help policy makers to ensure that problems of risk and uncertainty are properly addressed and mitigation strategies put into action before it is too late to avoid disastrous consequences?

The so-called precautionary principle [16] is a notion introduced by policy makers to evoke some kind of supreme "framework of thinking" in hazard assessment and management. It advocates the use of foresight in situations characterized by uncertainty and ignorance, where regulatory action as well as inaction could engender potentially large costs. A clear definition, however, does not exist. This lack of a clear terminology and pragmatic rules for due procedure, indicating what exactly needs to be done when and by whom as soon as early warning signals are detected, compounds the problems of complexity, uncertainty and controversy in risk management. Scientific uncertainty is far too often used as an excuse, to delay regulatory action on the one hand, and to foster public ignorance on the other. Who is to judge what risks can be considered acceptable, and who are the stakeholders that need to be represented in the decision making process? What exactly needs to be done when early warnings are issued, and how early should action be taken to protect vulnerable stakeholders from harm? The potential implications of early warnings may be quite clear to a scientific expert, a victim, a family doctor, a health inspector, or the members of an already exposed community, but how can we ensure that this knowledge is taken into account and communicated by those who have the power to act? There are no general ground rules for applying the precautionary principle. The communication and decision making quicksand at managerial levels, where priorities shift between the prevention of potentially harmful hazards and the promotion of potentially harmful economic priorities is badly in need of concrete rules which help dealing with situations of risk, uncertainty and ignorance in a responsible manner. 


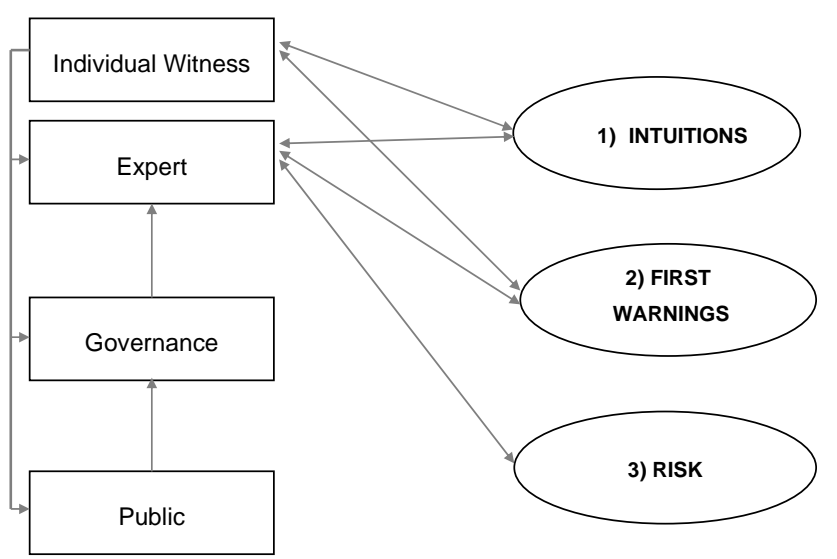

Fig. (4). The concept of uncertainty confounds three major levels of hazard cognition: 1) the first intuitions of a direct witness such as a family doctor, victim, expert or a small group of experts when nothing is known, 2) observations of witnesses or experts signaling early warnings when hazards may be suspected and 3) genuine risk knowledge implying that damages have already incurred and hazard statistics are available. The individual responsibility of all stakeholders to respect the communication contract is fully involved at all three levels.

The communication contract provides a few simple general rules for responsible information sharing in management, summarized here above in terms of ten ground clauses for information sharing and decision making. The original publication of this theory in the Journal of Business Ethics [4] gives a detailed account of the reasons why it is in the interest of all stakeholders to respect the ground clauses and to fully acknowledge individual responsibilities in the communication process. This can be directly applied to the particular case of disaster risk management on the basis of a stakeholder theory. Human intervention has engendered negative effects on the biosphere that are already deemed irreversible by experts. Some of them are likely to have hazardous long-term impacts at a global scale and therefore regulatory measures which ensure that early warnings are identified promptly and the relevant information is communicated effectively to stakeholders, are urgently need.

\section{A COMMUNICATION CONTRACT FOR DISASTER MANAGEMENT: WHAT IS AT STAKE AND WHO ARE THE STAKEHOLDERS?}

As soon as early warnings of disaster are detected, it becomes a matter of urgency to identify what is at stake and who the major stakeholders are. Respecting the communication clauses relative to relevance (2), clarity (6), continuity (7), balance (8), optimal timing (9) and prompt resolution (10) in information sharing and decision making is of essential importance here. While the prudence clause (3) commits stakeholders to careful consideration of uncertain information before action is precipitated, it must not ever be used as an excuse for non-action. Moreover, whenever early hazard warnings are issued and global and intangible long-term costs in terms of deaths and devastation of resources can be expected, as in the case of earthquakes, tsunamis, floods, and storms, which are mostly interdependent with potential consequences that are difficult to assess, preoccupations about false positives under the premise of a particular interpretation of the prudence clause must be dropped. This is a lesson mankind has already learnt from past experience and it should therefore guide all future decision making, as it is always better to be safe than sorry.

The concept of uncertainty in risk assessment confounds three functionally distinct levels of hazard cognition (Fig. 4): ignorance (no data about a hazard and its potential impacts are available, but individual observations and intuitions may be reported), uncertainty (impacts have been observed but predictive statistics are not available), and risk (impacts are known and statistics are available). Early warnings connect with the first or second level of hazard cognition. They may involve stakeholders at the individual level (direct witnesses) or the expert level (medical doctors, scientists, and other experts).

In the EEA case study on early warnings of asbestosis [18], it is reported that the earliest account of the health hazard represented by the blue dust particles were provided by an individual health inspector who was at the time not considered an expert, but who observed carefully, had the right intuitions and took the initiative to instigate further microscopic analyses of the dust, which confirmed her concerns. Despite the fact that the report was published and that experts had access to the knowledge made available, it was ignored. When the first action was triggered several decades later, asbestos induced mesothelomia had already reached epidemic proportions in the UK. Retrospectively, the delayed action has been explained by the fact that policy makers were placing short-term economic profits before the long-term interests of the workers and society. This is a clear example where the stakeholders who had the power to act and to protect the vulnerable ones from exposure did not manage their individual responsibility with the right amount of care by setting the right priorities. Some argue that such lack of individual responsibility in policy management can only be controlled by imposing heavy penalties on the wrongdoings of some, commensurate with the cost they inflict on others. Some argue for appropriate principles to guide individual action and governance to help meet society's long-term interests. This is where the communication contract finds its place. Ethical ground clauses for effective and transparent communication must be taken into account at all levels of hazard cognition, individual stakeholder responsibilities need to be clarified as early as possible.

At the level of knowledge where risks are real and hazard statistics available, it is often too late to implement successful mitigation strategies because the first losses have incurred and may already have triggered further consequences that cannot yet be predicted or even understood. The ground rules of the communication contract therefore apply from the first moment when intuitions of a direct witness such as a family doctor, a victim, or a small group of experts are reported. At that stage, it is important that individual witnesses take the responsibility to inform experts, and that experts inform the competent authorities immediately. Those who have the power to decide and act, have to make sure that the data are not ignored, but communicated to a larger and balanced group of independent experts, especially when little or nothing is still known about the hazard as such. Jargon is to 
be avoided, differences in expert viewpoints are to be fully respected and taken into account. All further communication involving the policy makers and the public is to respect the clauses of the contract to the best of knowledge and irrespective of hierarchical position or level of expertise. The major aim being to ensure that action can and will be taken as promptly as possible, the individual responsibility of all stakeholders is fully involved at any moment. How the communication contract can be implemented and monitored in responsible management or in crisis meetings is explained in greater detail in the original publication of the theory [4]. The general recognition of individual responsibility in this contract will ultimately lead to more accessible information from experts and science, increased awareness of a need for effective stakeholder participation at the decision making level, and a minimization of the long-term environmental and health costs to society.

Results from the initial case study, investigating business meetings where the communication contract was implemented in the decision making process of a small organization in crisis, have proven that a checklist and a short brief with minimal instructions suffice for putting it into practice in any group of individuals [4]. Group leaders or outside observers can monitor whether or not the different clauses of the contract are respected by individuals, and give feed-back to individuals and the group from meeting to meeting. It has been found that most individuals are at first totally unaware of their own clause violations as well as of those of the others, and found the briefing about these violations, and their potential negative influence on the communication process, very helpful. The communication contract thus initiates an individual and collective learning process, with progress that is quantitatively and qualitatively measurable from meeting to meeting, through the checklist and the briefs. It requires a minimum of instruction for monitoring, and therefore constitutes a simple yet powerful learning tool, in management training and elsewhere. The limitations of the tool and its underlying conceptual framework reside in the fact that it requires agreement and cooperation of all stakeholders involved. Also, the communication contract does not guarantee that increased awareness of responsibilities and consequences will indeed be followed by proper action, but it makes stakeholders think about the latter and their implications. Implementing the communication contract thereby creates optimal ground conditions for responsible and appropriate decision and action in due course [19]. This relates to the wider context of a class of theories relative to high reliability organizations [20], which argue that management learning needs to strive for high performance in situations that can be planned for, and for readiness to anticipate and adapt to unexpected events which, by definition, cannot be planned for.

\section{CONCLUSIONS}

Many of the losses caused by disasters officially reported, in the case studies cited here and in many others, could have been avoided by acting earlier. Expert knowledge of causes and their potential consequences is most often available a long time before action is taken, and cases of overreaction invoking so-called false positives have not yet been reported. The previous paragraphs of this paper have addressed some of the major reasons why successfully anticipating and managing risks requires awareness and responsibility of the experts, the governance, the exposed individuals and communities, and society as a whole. The communication contract introduces openness, clarity, relevance, balance, continuity and timing clauses for risk management aimed at maximizing the likelihood that the intuitions and experiences of individual observers, be they experts or not, are taken seriously by authorities and followed up by appropriate investigation and decision making. Adequate application of the prudence clause would consist of fully respecting early warnings based on low levels of scientific proof, which must not be dismissed on the grounds of motivations aimed at avoiding false positives. Responsibility must never solely rely on individuals with power to decide, but on committees with a broad panel of independent scientific experts and witnesses, as stipulated in the balance clause of the communication contract. While the latter cannot guarantee that no mistakes will be made, it provides an effective learning tool for increasing individual and collective awareness of responsibilities, and of the possible consequences of not assuming them effectively.

\section{REFERENCES}

[1] J-J. Rousseau, "The Social Contract", 1762 http://www.constitution.org/jjr/socon.htm

[2] T. Donaldson and T. W. Dunfee, "Towards a unified conception of business ethics: Integrative social contracts theory", Acad. Manag. Rev, vol. 19, no. 2, pp. 252-284, April. 1994.

[3] M. Dilley. Natural disaster hotspots: A global risk analysis. World Bank Press: Washington, 2005.

[4] B. Dresp-Langley, "The communication contract and its ten ground clauses", J Bus Ethics., vol. 87, no. 3, pp. 415-436, July, 2009.

[5] N.F. Pidgeon and R.E. Kasperson. "The social amplification of risk". Cambridge University Press: Cambridge, UK, 2003.

[6] C.T. Cheng, C.P. Ou and K.W. Chau,"Combining a fuzzy optimal model with a genetic algorithm to solve multiobjective rainfallrunoff model calibration," J. Hydrol., vol. 268, no. 1, pp. 472-86, Nov. 2002

[7] J.X. Xie, C.T. Cheng, K.W. Chau and Y.Z. Pei, "A hybrid adaptive time-delay neural network model for multi-step-ahead prediction of sunspot activity", Int. J. Environ. Pollut., vol. 28, no. 3/4 , pp. 364381, Feb. 2006.

[8] J.Y. Lin, C.T. Cheng and K.W. Chau, "Using support vector machines for long-term discharge prediction," J. Hydrol. Sci., vol. 51, no. 4, pp. 599-612, Jan. 2006.

[9] M.Y. Zhao, C.T. Cheng, K.W. Chau and G. Li, "Multiple criteria data envelopment analysis for full ranking units associated to environment impact assessment", Int. J. Environ. Pollut., vol. 28, no. 3, pp. 4448-464, Oct. 2006.

[10] N. Muttil and K.W. Chau, "Machine learning paradigms for selecting ecologically significant input variables", Eng. Appl. Artif. Intel., vol. 20, no. 6, pp. 735-744, Jan. 2007.

[11] K.W. Chau, "Particle swarm optimization training algorithm for ANNs in stage prediction of Shing Mun River", J. Hydrol., vol. 329 , no. $3 / 4$, pp. $363-367$, Oct. 2006.

[12] J. L. Austin. How to do things with words. Oxford University Press: Oxford, UK, 1962.

[13] J. Searle, Speech Acts: An Essay in the Philosophy of Language. Cambridge University Press: Cambridge, 1969.

[14] T. Reid, "Essays on the Active Powers of the Human Mind: Inquiry into the Human Mind on the Principles of Common Sense", Kessinger Publishing: Whitefish, MT, 1843.

[15] A. Bandura, "Social cognitive theory: An agentic perspective", Annu. Rev. Psychol., vol. 52, no. 1, pp. 1-26, Feb. 2001.

[16] The European Environment Agency, "Late lessons from early warnings: The precautionary principle 1896-2000”, EEA Publications: Copenhagen, 2001. http://www.eea.eu.int 
[17] L. Edqvist and K. B. Pedersen, "Antimicrobials as growth promoters: resistance to common sense", Copenhagen: EEA Publications: Copenhagen, 2001, pp. 93-99. http://www.eea.eu.int

[18] D. Gee and M. Greenberg, "Asbestos: from 'magic' to malevolent mineral", EEA Publications: Copenhagen, 2001, pp. 5263.http://www.eea.eu.int
[19] J. Baron. "Thinking about consequences", J. Moral. Educ., vol. 19, no.2, pp. 77-87, April 1990.

[20] K.E. Weick and K.M. Sutcliffe. "Managing the unexpected: Resilient performance in an age of uncertainty". Jossey-Bass: San Francisco, 2007.

Received: January 10, 2012

Revised: February 01, 2012

Accepted: February 10, 2012

(C) Dresp-Langley et al.; Licensee Bentham Open.

This is an open access article licensed under the terms of the Creative Commons Attribution Non-Commercial License (http://creativecommons.org/licenses/by-nc/3.0/g) which permits unrestricted, non-commercial use, distribution and reproduction in any medium, provided the work is properly cited. 